\title{
Nitric oxide (NO) signaling in Trichoplax and related species: Microchemical characterization and the lineage-specific diversification
}

Leonid L. Moroz ${ }^{11 \%}$, Daria Y. Romanova ${ }^{2,4 \pi}$, Mikhail A. Nikitin ${ }^{3 \pi}$, Dosung Sohn ${ }^{1 \pi}$, Andrea B. $\mathrm{Kohn}^{1, \text {,I }}$, Emilie Neveu ${ }^{4}$, Frederique Varoqueaux ${ }^{4 *}$, Dirk Fasshauer ${ }^{4}$

${ }^{1}$ Whitney Laboratory for Marine Biosciences and Departments of Neuroscience, University of Florida, St. Augustine and Gainesville, FL, 32080, USA; ${ }^{2}$ Institute of Higher Nervous Activity and Neurophysiology, Russian Academy of Sciences, Moscow 117485, Russia; ${ }^{3}$ Belozersky Institute of Physico-Chemical Biology, Moscow State University, Moscow 119991, Russia; ${ }^{4}$ Department of Fundamental Neurosciences, University of Lausanne, 1005 Lausanne, Switzerland

* Corresponding authors

Emails: moroz@whitney.ufl.edu

https://orcid.org/0000-0002-1333-3176

http://orcid.org/0000-0002-1040-4282

IThese authors contributed equally to this work.

Acknowledgments: Acknowledgments: This work was supported by the Human Frontiers Science Program (RGP0060/2017), National Science Foundation (1146575, 1557923, 1548121 and 1645219) grants to L.L.M; and the Swiss National Science Foundation (\#31003A_182732) to D.F. 


\begin{abstract}
:
Nitric oxide (NO) is a free radical gaseous messenger with a broad distribution across the animal kingdom. However, the early evolution of nitric oxide-mediated signaling in animals is unclear due to limited information about prebilaterian metazoans such as placozoans. Here, we analyzed NO synthases (NOS) in four different species of placozoans (haplotypes H1, H2, H4, H13). In contrast to all other invertebrates studied, Hoilungia and Trichoplax have three distinct NOS genes, including PDZ domain-containing NOS. To characterize NOS activity in Trichoplax adhaerens, we used capillary electrophoresis for microchemical assays of NO-related metabolites. Specifically, we quantified nitrites (products of NO oxidation) and L-citrulline (co-product of NO synthesis from L-arginine), which were affected by NOS inhibitors confirming the presence of functional NOS. Next, using fluorescent single-molecule in situ hybridization, we showed that distinct NOSs are expressed in different subpopulations of cells, with a noticeable distribution close to the edge regions of Trichoplax. These data suggest the compartmentalized release of this messenger and a greater diversity of cell types in placozoans than anticipated. We also revealed a dramatic diversification of NO receptor machinery, including identification of both canonical and novel NIT-domain containing soluble guanylate cyclases as putative NO/nitrite/nitrate sensors. Thus, although Trichoplax is considered to be one of the morphologically simplest free-living animals, the complexity of NO-cGMP-mediated signaling is greater to those in vertebrates. This situation illuminates multiple lineage-specific diversifications of NOSs and NO/nitrite/nitrate sensors from the common ancestor of Metazoa.
\end{abstract}

Keywords: Placozoa, basal Metazoa, Trichoplax, Hoilungia, the evolution of nitric oxide signaling, capillary electrophoresis, nitrites, L-citrulline, in situ hybridization, NIT domain, cGMP, ctenophores. 


\section{Short Abstract}

Nitric oxide (NO) is a ubiquitous gaseous messenger, but we know little about its early evolution. Here, we analyzed NO synthases (NOS) in four different species of placozoans - one of the earlybranching animal lineages. In contrast to other invertebrates studied, Trichoplax and Hoilungia have three distinct NOS genes, including PDZ domain-containing NOS. Using ultra-sensitive capillary electrophoresis assays, we quantified nitrites (products of NO oxidation) and L-citrulline (co-product of NO synthesis from L-arginine), which were affected by NOS inhibitors confirming the presence of functional enzymes in Trichoplax. Using fluorescent single-molecule in situ hybridization, we showed that distinct NOSs are expressed in different subpopulations of cells, with a noticeable distribution close to the edge regions of Trichoplax. These data suggest both the compartmentalized release of $\mathrm{NO}$ and a greater diversity of cell types in placozoans than anticipated. NO receptor machinery includes both canonical and novel NIT-domain containing soluble guanylate cyclases as putative NO/nitrite/nitrate sensors. Thus, although Trichoplax and Hoilungia exemplify the morphologically simplest free-living animals, the complexity of NOcGMP-mediated signaling in Placozoa is greater to those in vertebrates. This situation illuminates multiple lineage-specific diversifications of NOSs and NO/nitrite/nitrate sensors from the common ancestor of Metazoa. 


\section{Introduction}

Nitric oxide (NO) is a versatile gaseous transmitter widely distributed among prokaryotes and eukaryotes ${ }^{1-4}$. Multiple functions of this messenger are direct reflections of the free-radical nature of $\mathrm{NO}$ and, subsequently, its complex free radical chemistry ${ }^{5}$. Dissolved NO passes readily across membranes and diffuses into neighboring cells interacting with many biological molecules including DNA, lipids, proteins ${ }^{5}$ with several specialized receptors such as guanylate cyclases ${ }^{6-8}$. Thus, NO can act as a volume transmitter locally, and it is easily converted into nitrite and nitrate radical by oxygen and water. In cells, NO is catalyzed by the enzyme NO synthase (NOS) through a series of complex redox reactions by the deamination of the amino acid Larginine to L-citrulline. The reaction requires the presence of oxygen as a precursor and NADPH ${ }^{5}$. The large enzyme operates as a dimer and consists of two enzymatic portions, an oxygenase domain that binds heme and the redox factor tetrahydrobiopterin $(\mathrm{H} 4 \mathrm{~B})$ and a reductase domain that is related to NADPH-dependent microsomal cytochrome $\mathrm{P} 450{ }^{9}$.

The role and mechanism of NO signaling are well studied in mammals. However, little is known about the early evolution of NO signaling in animals, mostly due to limited comparative data from basally branching metazoans, including Cnidaria, Porifera, Ctenophora, and Placozoa.

Among other things, NO is involved in feeding, chemosensory processing, and locomotion of such cnidarians as Hydra and Aglantha 10-13, where NO-dependent communications were likely mediated by just one type of NO synthase (NOS) ${ }^{1}$. In the sponge Amphimedon, only one NOS gene has been identified ${ }^{14}$. NO-cGMP signaling has been implemented in the regulation of larval settlement ${ }^{15}$ and rhythmic body contractions
16. In the ctenophore, Mnemiopsis leidyi, again, only one NOS gene has been recognized so far ${ }^{17}$, but the functional role of NO has not been studied. Interestingly, in another ctenophore species, Pleurobrachia bachei, NOS appears to have been lost ${ }^{18}$.

Nothing is known about the presence and the distribution of $\mathrm{NO}$ signaling in Placozoa - an important but little-studied lineage of cryptic marine animals. The current consensus stands that Placozoa is the sister group to the clade Cnidaria+Bilarteria ${ }^{18-20}$, although some authors consider Placozoa as highly derived and secondarily simplified cnidarians ${ }^{21}$. Regardless of the proposed phylogenies, Placozoa represents a crucial taxon to understand the origin and evolution of animal traits and the nervous system in particular ${ }^{22}$.

Placozoans, such as Trichoplax and their kin ${ }^{23}$, are the simplest known freeliving animals with only six morphologically recognized cell types organized in three layers ${ }^{24}$. Nevertheless, Trichoplax has quite complex behaviors ${ }^{25-28}$, including social-like patterns ${ }^{29}$. Here, we biochemically showed that Trichoplax exhibits functional NOS activity, and, in contrast to other prebilaterian animals, placozoa independently evolved three distinct NOSs (as vertebrates) with a profound diversification of NO-cGMP signaling components, and likely the capabilities of nitrite/nitrate sensing by distinct NIT domain-containing guanylyl cyclases, which represents a remarkable example of the evolution of gaseous transmission in the animal kingdom.

\section{Materials and Methods}

Animals and culturing. Trichoplax adhaerens (H1 haplotype) and Hoilungia hongkongensis (H13 haplotype), 0.3-2 mm in diameter, were maintained in the laboratory 
culture as described elsewhere, and animals were fed on rice grains and algae ${ }^{24,30}$.

Direct microchemical assays of NOS metabolites such as $\mathrm{NO}_{2}^{-}$, L-arginine, Lcitrulline were performed using highresolution capillary electrophoresis (CE) with both conductivity and laser-induced fluorescence (LIF) detectors. The principles and details of major protocols for NOS activity assay were reported ${ }^{31-33}$ with some minor modifications. We made minor adjustments to these protocols, which we briefly summarize below.

Nitrite/Nitrate Microanalysis using CE with Contactless Conductivity. CE, coupled with a TraceDec contactless conductivity detector (Strasshof, Austria) was used for the assay of nitrite and nitrate. To reduce $\mathrm{Cl}^{-}$in a sample, we used OnGuard II Ag (DIONEX Corp., Sunnyvale, CA). We used custom-built cartridges for small volume $(20 \mu \mathrm{L})$ sample clean-up by a solidphase extraction technique as reported ${ }^{34}$. In brief, 4 5 $\mathrm{mg}$ of the resin was backloaded in a $10 \mu \mathrm{L}$ filter-pipette tip, and the microcartridge was washed with $1 \mathrm{~mL}$ of ultrapure water using a $3 \mathrm{~mL}$ disposable syringe. The pre-washed cartridge was put into a $200 \mu \mathrm{L}$ pipette tip to avoid surface contamination during further centrifugation. Extra water remaining in the cartridge was removed by centrifugation at $1000 \mathrm{rpm}$ for 30 seconds. Then, the assembly was inserted into a 0.5 mL PCR tube, and a final diluted sample was loaded into the preconditioned cartridge followed by centrifugation at $1000 \mathrm{rpm}$ for 30 seconds, causing the sample to pass through the silver resin. To quantitate any potential sample loss, the custom-made chloride cartridge was tested for sample recovery of both nitrite and nitrate.

All experiments were conducted using a $75 \mathrm{~cm}$ length of $50 \mu \mathrm{m}$ I.D. $\times 360 \mu \mathrm{m}$ O.D. fused silica capillary (Polymicro Technologies, AZ) with an insulated outlet conductivity cell. Arginine/borate electrolyte was used for a separation buffer with tetradecyltrimethylammonium hydroxide (TTAOH) added as an EOF modifier. The modifier was prepared from tetradecyltrimethylammonium bromide (TTABr) by an OnGuard-II A cartridge (DIONEX Corp., CA) treated with $1 \mathrm{M}$ $\mathrm{NaOH}$. For separation steps, the capillary inner-wall was successively washed with $1 \mathrm{M}$ $\mathrm{NaOH}$, ultrapure water, and the separation buffer (25 mM Arg, $81 \mathrm{mM}$ Boric acid, and $0.5 \mathrm{mM}$ TTAOH, $\mathrm{pH}$ 9.0) by applying pressure (1900 mbar) to the inlet vial. Since nitrite and nitrate concentrations were very low in diluted samples, capillary isotachophoresis (CITP), a sample stacking method, was employed. The leading solution was introduced into the capillary by pressure injection (25 mbar for 12s), and then a neuronal sample was loaded using electrokinetic injection $(-5 \mathrm{kV}$ for $12 \mathrm{~s})$. The separation was performed under a stable $15 \mathrm{kV}$ voltage at $20^{\circ} \mathrm{C}$.

\section{Amino Acids Microanalysis using} CE with laser-induced fluorescence detection. The $\mathrm{CE}$, coupled with the ZETALIF detector (Picometrics, France), was used for the assay of amino acids. In this work, a helium-cadmium laser $(325 \mathrm{~nm})$ from Melles Griot, Inc. (Omnichrome® Series56, Carlsbad, CA) was used as the excitation source. Before the photomultiplier tube (PMT), the fluorescence was both wavelengths filtered and spatially filtered using a machined 3-mm pinhole. All instrumentation, counting, and high-voltage CE power supply were controlled using DAx 7.3 software.

All solutions were prepared with ultrapure Milli-Q water (Milli-Q filtration system, Millipore, Bedford, MA) to minimize the presence of impurities. Borate buffer (30 mM, pH 9.5) was used for sample preparation. All solutions were filtered using 
$0.2 \mu \mathrm{m}$ filters to remove particulates. The buffers were degassed by ultrasonication for 10 min to minimize the chance of bubble formation. A $75 \quad \mathrm{mM} \quad \mathrm{OPA} / \beta$ mercaptoethanol $(\beta-\mathrm{ME})$ stock solution was prepared by dissolving $10 \mathrm{mg}$ of OPA in 100 $\mu \mathrm{L}$ of methanol and mixing with $1 \mathrm{~mL}$ of 30 $\mathrm{mM}$ borate and $10 \mu \mathrm{L}$ of $\beta$-ME. Stock solutions $(10 \mathrm{mM})$ of amino acids were prepared by dissolving each compound in the borate buffer. OPA and $\beta$-ME were stored in a refrigerator, and fresh solutions were prepared weekly.

All experiments were conducted using a $75 \mathrm{~cm}$ length of $50 \mu \mathrm{m}$ I.D. $\times 360 \mu \mathrm{m}$ O.D. fused silica capillary (Polymicro Technologies, AZ). A $30 \mathrm{mM}$ borate/30 mM sodium dodecyl sulfate (SDS) electrolyte (adjusted to $\mathrm{pH} 10.0$ with $\mathrm{NaOH}$ ) was used as the separation buffer for amino acid analysis. The pre-column derivatization method was used. A $1 \mu \mathrm{L}$ of $o$-Phthalaldehyde (OPA) was incubated in a $0.5 \mathrm{~mL}$ PCR tube. The total volume of a sample, OPA, and internal standard inside the tube was $20 \mu \mathrm{L}$. For separation steps, the capillary inner-wall was successively washed with $1 \mathrm{M} \mathrm{NaOH}$, Milli Q water, and the separation buffer by applying pressure (1900 mbar) to the inlet vial. Then the sample was loaded using electrokinetic injection ( $8 \mathrm{kV}$ for $12 \mathrm{~s}$ ). The separation was performed under a stable $20 \mathrm{kV}$ voltage at $20^{\circ} \mathrm{C}$.

In all CE tests, once an electropherogram was acquired, peaks were assigned based on the electrophoretic mobility of each analyte, and the assignments were confirmed by spiking corresponding standards into the sample. Five-point calibration curves (peak area vs. concentration) of analytes were constructed for quantification using standard solutions. All chemicals for buffers were obtained from Sigma-Aldrich, and standard amino acids were purchased from Fluka. Ultrapure Milli-
Q was used for all solutions and sample preparations.

NOS inhibitors' tests. To establish that NOS enzymatic activity is responsible for producing the Arg/Cit ratio and nitrite measured in Trichoplax, the whole animal was incubated in one of NOS inhibitors (e.g., $N^{\mathrm{G}}$-nitro-1-arginine methyl ester (L-NAME); besides, another NOS inhibitor, L-N ${ }^{6}-(1-$ iminoethyl)-lysine (L-NIL), showed very effective inhibition as in molluscan preparations ${ }^{35}$.

After the animals were isolated from the culture medium, they were placed in a 0.5 $\mathrm{mL}$ PCR tube and incubated with certain concentrations of NOS inhibitors for 30 minutes at room temperature, followed by washing with artificial seawater. Then, all the water was removed, and $1 \mu \mathrm{L}$ of Milli Q water was dropped onto the animal, and the tube was stored at $-80^{\circ} \mathrm{C}$ until use.

Specifically, we also performed a series of control tests to see if there were any small molecules that might interfere with peak identifications. Water, L-NAME, and L-NIL controls were first tested, and no nitrite was observed. However, chloride and nitrate ions were always present, because all NOS inhibitors contain chloride, and nitrate is a common impurity in most of the commercially used chemicals. Fresh single individuals of Trichoplax by itself, and Trichoplax incubated with NOS inhibitors were then analyzed. An effective NOS inhibitor should cause the nitrite level to be lower than in the animal compared to control tests.

Comparative bioinformatic analyses. We used the data from the sequenced genomes of two sequenced placozoan species ${ }^{36,37}$, and our additional sequencing data are presented in the supplement 1 . 
Protein sequences were aligned in MUSCLE ${ }^{38}$. Phylogenetic trees were inferred using Maximum Likelihood algorithm implemented in IQTREE web server http://iqtree.cibiv.univie.ac.at/, ${ }^{39}$. Tree robustness was tested with 2000 replicates of ultrafast bootstrap ${ }^{40,41}$.

To test for positive and negative selection, the following algorithms were used: codon-based Z-test and Fischer's exact test implemented in MEGA $\mathrm{X}^{42-44}$, and ABSREL, BUSTED, FUBAR and MEME in HyPhy package ${ }^{45-49}$. Evolutionary distances were calculated in MEGA $X$ under the Poisson method and gamma-distributed rates across sites.

\section{Fixative-resistant}

NADPH-

diaphorase activity has been widely used as a histochemical reporter of NOS in both vertebrates and invertebrates ${ }^{33,50-53}$. Thus, we used this approach to screen for putative NOS activity in Trichoplax and Hoilungia. All methodological details of the protocol have been described earlier ${ }^{54-57}$, and we used 45 and $90 \mathrm{~min}$ fixation in $4 \%$ freshly made paraformaldehyde solution made using the filtered seawater.

In situ hybridization was performed using the RNAscope multiplex fluorescent Reagent kit v2 assay (Advanced Cell Diagnostics, Inc, Bio-Techne, USA) as specified by the company protocol (https://acdbio.com/rnascope\%C2\%AE-

multiplex-fluorescent-v2-assay). In brief, we transferred 10-15 animals to the glass slides with cavities with $2 \mathrm{~mL}$ fresh $0.2 \mu \mathrm{m}$ filtered seawater, washed three times, and removed the seawater under a microscope. Next, we fixed animals using $4 \%$ paraformaldehyde in seawater for $30 \mathrm{~min}$ at room temperature, performed dehydration and rehydration steps with increased and decreased concentrations of ethanol $(30 \%, 50 \%, 70 \%, 100 \%$ on PBS) at room temperature. We pretreated animals in Protease III (Sigma) for $10 \mathrm{~min}$ at room temperature. The rest of the protocol is reported elsewhere (Advanced Cell Diagnostics, ACD \#323110 at web site https://acdbio.com/rnascope\%C2\%AEmultiplex-fluorescent-v2-assay). The key point in the procedure is to use tyramide signal amplification steps to detect low abundant genes as NOSs.

For all imaging, we used fluorescent microscope Nikon Ti2 (Nikon, Japan) with a spinning disk (Crest Optics X-Light V2).

\section{Results and Discussion}

\section{Comparative analysis of NOSs}

Fig. 1 shows the genealogical relationships among different animal NOSs, where representatives of all basal metazoan lineages form distinct branches for their respected NOSs with evidence for relatively recent duplication events consistent to an early origin and diversification of NOSs in other eukaryotic groups including Amebozoa and Fungi as sister lineages to Metazoa. We did not find the evidence for NOS in choanoflagelates sequenced so far, including Monosiga with the sequenced genome. Choanozoa, the phylogenetically closest taxon to Metazoa ${ }^{58}$, might have lost NOS from its eukaryotic ancestor. Interestingly, ctenophores, known as the sister lineage to the rest of metazoans ${ }^{19,20,59}$, have only one highly derived NOS as represented by two Mnemiopsis species and Cestum in the tree (Fig. 1).

All studied invertebrates have only one or two NOS genes, which do not directly correspond to the well-established vertebrate subfamilies of the enzymes ${ }^{1}$. In contrast, we identified three distinct NOS genes in the Trichoplax genome (haplotype H1), and three other placozoan species or haplotypes $\mathrm{H} 2, \mathrm{H} 4, \mathrm{H} 13$, and one of the NOSs contains 
the PDZ domain similar to the mammalian neuronal NOS.

The presence of PDZ domaincontaining NOSs is a distinct feature of all four placozoan species sequenced so far (NOS1 in $\mathrm{H} 1, \mathrm{H} 2, \mathrm{H} 4$, and $\mathrm{H} 13$ ). $\mathrm{H} 1$ and $\mathrm{H} 2$ represent the classical Trichoplax genus ${ }^{23}$, while $\mathrm{H} 4$ and $\mathrm{H} 13$ belong to the newly described genus Hoilungia ${ }^{36,60}$. The clustering of NOSs in placozoans reflects their phylogenetic relationships stressing that $\mathrm{H} 4$ and H13 (Hoilungia) vs. $\mathrm{H} 1$ and $\mathrm{H} 2$ (Trichoplax) belong to different lineages.

The PDZ domain and N-terminal motifs are required for the anchoring of NOS to plasmatic or intracellular membranes, subcellular localization, and integration to many signaling components like in the mammalian neuronal nNOS ${ }^{9,61-64}$. nNOS is different from the two other mammalian isoforms as its N-terminal PDZ domain can heterodimerize with the PDZ domains of PSD95 or syntrophin ${ }^{65}$ and others ${ }^{9}$. Thus, we might suggest similar molecular functions in Trichoplax and Hoilungia.

The rate of evolution of the PDZ domain-containing NOSs is comparable to other NOSs for all placozoan species. The branching patterns of NOS trees (Fig. 1) reveals that three NOSs in Placozoa are the results of two independent duplication events from the common placozoan ancestor. The first splitting separated NOS1, and the second, more recent split led to NOS2 and NOS3.

Of note, we also identified two NOSs in the stony coral Stylophora, which has one NOSs with the PDZ domain (Fig. 1), and a PDZ domain was detected upstream of the Nematostella NOS gene. Also, two sponges (Amphimedon and Spongilla) possess PDZcontaining NOS. As the PDZ domains of NOSs appear to be homologous, it should be investigated whether the PDZ domain- containing form represents the ancestral form in animals. In contrast, ctenophores and many other animal lineages (Fig. 1) do not have PDZ-containing NOS genes.

NOS is a complex enzyme requiring several co-factors for its activation, and $\mathrm{Ca}^{2+}$ dependence of different NOSs in mammals is determined by the presence of the autoinhibitory inserts and calmodulinbinding sites 66-73. Fig. 2 shows the presence/absence of such motifs and the auto-inhibitory loops across basal metazoan lineages. The canonical human $\mathrm{Ca}^{2+}$ independent iNOS lacks such a loop; it is bound to $\mathrm{CaM}$ in a $\mathrm{Ca}^{2+}$-independent manner. Mammalian iNOS activation is often induced by lipopolysaccharides as a part of innate immunity responses on bacterial infection ${ }^{4}$. Ctenophore, the demosponge Amphimedon, Nematostella, and three coral NOSs also lack the auto-inhibitory loop (Fig. 2) and could be $\mathrm{Ca}^{2+}$-independent and, apparently, inducible (e.g., by bacteria or during development and differentiation). However, all three Trichoplax and Hoilungia NOSs contains an intermediate size insert in this position: these NOSs might be dormant or, at least, partially inducible. Thus, the direct detection of endogenous enzymatic activity is needed to validate NOS expression, which we performed using direct microchemical assays.

\section{Detection of endogenous NOS activity in Trichoplax}

Because some NOSs can be inducible or pseudogenes, the molecular/sequence information itself is not sufficient for the demonstration of NOS activity. Thus, we implement two complementary approaches to confirm the presence of functional NOSs in placozoans.

Arginine/citrulline assays. NO is known to be produced enzymatically from molecular 
oxygen and L-arginine with L-citrulline as the co-product ${ }^{5}$. Using a highly sensitive capillary electrophoresis $(\mathrm{CE})$ microchemical assay with attomole detection limits, we demonstrated that Trichoplax produced Lcitrulline, and its production is also eliminated by NOS inhibitors (Fig. 3). It was expected from experiments on vertebrates and mollusks $32,33,35$ that the arginine-tocitrulline ratio would increase after Trichoplax was incubated in either LNAME/D-NAME or L-NIL. the arginine-tocitrulline ratio increased by two-fold in the case of L-NIL (Fig. 3). However, there was only a small increase with L-NAME, indicating L-NIL effectively inhibited the NOS enzyme as in mollusks ${ }^{32,33,35}$, but LNAME did not. The reason for this difference might reflect differences in either L-arginine uptake, which might be blocked by arginine analogs or distinct enzymatic regulation of NOS in placozoans, or nonenzymatic interference of these inhibitors with $\mathrm{NO}$ production ${ }^{74}$.

It was interesting that all NOS-related metabolites were detected in Trichoplax at relatively high concentrations, $0.35 \mathrm{mM}$ for arginine and $0.5 \mathrm{mM}$ for citrulline. Combined, these $\mathrm{CE} /$ microchemical data indicate that placozoans have a substantial level of endogenous NOS activity.

Nitrite assays. Due to rapid NO oxidation in biological tissues ${ }^{5}, \mathrm{NO}_{2}{ }^{-}$is considered as the most reliable reporter of functional NOS. In contrast, more stable (and less dynamic) terminal oxidation products of $\mathrm{NO}$ - nitrates $\left(\mathrm{NO}_{3}{ }^{-}\right)$cannot be used for these purposes since they can also be accumulated from various food sources. Thus, by employing $\mathrm{CE}$ with the conductivity detection, we provided the additional direct evidence for endogenous NOS activity using nitrite $\left(\mathrm{NO}_{2}{ }^{-}\right)$assay ${ }^{31,33}$.

NO oxidation metabolites were monitored, and concentrations were derived from in vitro calibration curves prepared from standard solutions of nitrate and nitrite at various concentrations $(10 \mathrm{nM}-500 \mu \mathrm{M})$. With the regression equations, the limit of detection (LOD) of nitrate was determined to be $13.3 \mathrm{nM}$ for nitrite and $32.4 \mathrm{nM}$ for nitrate. These LODs were sufficient to quantify nitrite and nitrate in Trichoplax.

Surprisingly, we found than Trichoplax contains millimolar concentrations of $\mathrm{NO}_{2}{ }^{-}$and $\mathrm{NO}_{3}{ }^{-}$, which were eliminated, within $30 \mathrm{~min}$, by NOS inhibitors such as L-NAME and L-NIO (Fig. 4). In control Trichoplax, about $1.5 \mathrm{mM}$ nitrite was detected, but after incubated with the NOS inhibitors, no nitrite was observed, suggesting the suppression of endogenous NOS activity (Fig. 4).

The expression and distribution of NOS in Trichoplax

Fixative-resistant NADPHdiaphorase (NADPH-d) histochemistry has been reported as a marker of functional NOS in both vertebrates and invertebrates ${ }^{33,50-53}$. Here, we employed this assay for the initial screening of the NOS expression in Trichoplax adhaerens (H1) and its related species Hoilungia hongkongensis ${ }^{36}$. The NADPH-d histochemical activities in both placozoans were significantly weaker compared to the majority of other species studied using the same protocol 13,54,55,57,75-77. We noted that the intensity of NADPH-d labeling was similar to those described in the pelagic pteropod mollusk Clione limacina, where NO controlled swimming ${ }^{56}$.

We revealed very similar NADPH-d labeling patterns in both Trichoplax and Hoilungia (Fig. 5A, B). There were several large $(>10 \mu \mathrm{m})$ structures; some of them correspond to the so-called "shinny spheres" 78 and numerous small (4-6 $\mu \mathrm{m})$ NADPH-d reactive cells were broadly distributed over 
different parts of the animal including the dorsal epithelial layer. We estimate that about $2 \%$ of placozoan cells might be NADPH-d reactive. These cells might be candidates for NOS-containing (NO-releasing) cells. However, NADPH-d histochemistry cannot distinguish different NOS isoforms.

\section{Single-molecule in fluorescent in situ hybridization (FISH)}

Next, we used sequences for both NOS1 and NOS3 to characterize their expression and distribution in Trichoplax adhaerens by single-molecule FISH as the most sensitive assay for this purpose. In both cases, we observed the cell-specific distribution of distinct NOS isoforms (Fig. 5C). Most of the NOS-containing cells were broadly distributed (similar to NADPH-d reactivity, but "shiny spheres" were not labeled by in situ hybridization probes). It appears that PDZ containing NOS1 expressed in more cells than NOS3, and only partial co-localization of the two NOSs was observed (Fig. 5C). We also noted that the NOSs are not located to the most peripheral cell layer but found in cells close to the edge. Due to a relatively high level of endogenous fluorescence in the central part of the animal, the precise cell identity of NOS-positive cells was difficult to determine. However, we noticed that both NOS could be co-localized in a very small subset of cells close to the edge of these disk-like animals.

\section{NO targets and diversification of cGMP signaling in Placozoa}

NO can act via cyclic guanosine monophosphate or cGMP as second messenger. In this signaling pathway, NO binds to the heme group of soluble guanylate cyclases (sGCs), member of the adenylyl cyclase superfamily ${ }^{79,80}$, with a characteristic catalytic CYC domain, leading to the increase of cGMP synthesis ${ }^{6-8,81-85}$; by binding to ATP, sGC can also couple NO signaling to cellular metabolism ${ }^{86}$.

Surprisingly, the Trichoplax and Hoilungia genomes encode seven sGCs (Fig. 6A), whereas only three orthologs were identified in humans. All these enzymes have the canonical heme NO binding domain and associated domain organization, and the predicted sGCs from placozoans from clusters appropriately with the $\alpha$ and $\beta$ sGCs of humans.

We identified in Trichoplax and their kin additional membrane-bound NO receptor candidates (Fig. 6A). Trichoplax also has five orthologs of atrial natriuretic peptide-like receptors (ANPRs) with CYC/cGMP coupling as in humans. But there is no atrial natriuretic peptide detected in any sequenced placozoan genome. There are also four Trichoplax adenylate cyclases, which have two CYC domains (humans have nine adenylate cyclases); these are probably not involved in NO binding and we used them as outgroups.

Unexpectedly, we discovered 12 additional guanylyl cyclases with unique NIT domains ${ }^{87}$, which were only previously known from bacteria as nitrate and nitrite sensors 88,89 . Nitrate/nitrite sensing type domain in placozoans (NIT: PF08376) is flanked between two transmembrane domains and a $\mathrm{C}$-terminal guanylate cyclase catalytic domain (AC/GC: PF00211). The same critical amino acid residues that were observed in the bacterial sequences were also present in the predicted placozoan NIT domains. (Fig. 6B). The tree shows clearly that they belong to the ANPR type/group and probably arose by lateral gene transfer into an existing ANPR type, which is established as guanylate cyclase. 
The heme-dependent NO sensor HNOBA (PF07701) is also found associated with some of these predicted proteins.

To the best of our knowledge, these types of NIT containing proteins have not been previously characterized in animals. There is no NIT domain detected in the sequenced genomes of ctenophores and sponges. However, the observed NIT abundance in placozoans suggests potential sensing of nitrites and/or nitrates. This hypothesis is consistent with our present finding of the micromolar concentration of nitrites in Trichoplax. Because many placozoan cells (e.g., fiber cells) do contain endosymbiotic bacteria, additional levels of intra- and intercellular NO-dependent communications are also highly likely and can be tested in future studies.

Even more interesting, we found NIT-containing GCs across bilaterians including molluscs, annelids, arthropods, priapulids, echinoderms, hemichordates and basal chordates but vertebrates apparently lost NIT domains (Fig. 2 Supplement). Apparently, molluscs, hemichordates (Saccoglossus) and placozoans have one of the largest numbers of predicted NIT domain genes compared to all studied metazoans.

The model cnidarian Nematostella has no NIT domain, but there are NITcontaining genes in the genome of related anthozoan species including corals. The supplementary phylogenetic tree shows that all metazoan NIT-GCs cluster together and their NIT domains are more similar to each other than to bacterial NITs.

The exact function of the NIT domain in animals is yet to be elucidated, but the same architectural domain organization of the NIT domain ${ }^{89,90}$ is observed across metazoans (Fig. 6B) inferring a similar function. In bacteria, it has been proposed that the NIT domain regulates cellular functions in response to changes in nitrate and/or nitrite concentrations, both extracellular and intracellular ${ }^{88,89}$. The same possibility of nitrite/nitrate sensing might be widespread across the animal kingdom. Functional studies would be needed to carefully test this hypothesis in the future. 


\section{Highlights:}

1. The phylogenetic position of Placozoa, as an early branching metazoan lineage, and the simplicity of morphological organization emphasizes the importance of Trichoplax as one of the key reference species for understanding the origin and evolution of animals and their signaling mechanisms ${ }^{91}$, including NO-/cGMP-mediated signaling.

2. Our combined genomic, molecular, and microchemical analyses strongly indicate the presence of functional NOSs in Trichoplax, which is broadly distributed across different cell populations. In contrast to other prebilaterian animals, placozoans independently evolved three different NOS genes, similar to the situation in vertebrates. This relatively recent diversification of enzymes producing gaseous free radical messenger illustrates the parallel development of complex signaling mechanisms in placozoans and implies a much greater complexity of intercellular communications than it was anticipated before.

3. The molecular targets of NO in Trichoplax can be seven soluble guanylyl cyclases (sGCs) and five membrane-bound ANP-like receptors. Besides, we identified at least twelve cyclases with unique NIT domains. Placozoans have the largest number of predicted NIT domain genes compared to all studied metazoans. We hypothesize that in placozoans, as in bacteria, the putative NIT domain is used as nitrate/nitrite-sensing due to the high levels of nitrate/nitrites measured in Trichoplax.

In summary, although canonical functional NO-cGMP signaling could be a highly conservative feature across Metazoa, the enormous diversity of molecular components of these and related pathways in placozoans stress the cryptic complexity of these morphologically simplest animals.

\section{Acknowledgments}

We thank the Cellular Imaging Facility (UNIL, Lausanne, Switzerland) for FHL for their excellent support and Friday Harbor Laboratories for their support to collect animals.

\section{Role of authors}

All authors had access to the data in the study and take responsibility for the integrity of the data and the accuracy of the data analysis. ABK, DYR and MAN share authorship equally. Research design: LLM. Acquisition of data: all authors (Molecular data and sequencing analyses ABK, MAN, DYR, EN, DF, LLM; in situ hybridization FV, DYR, LLM, DF; Microchemical assays: LLM and DS; NADPH-d: DYR, LLM). Analysis and interpretation of data: all authors. Drafting of the article: LLM. Funding: LLM, DF. 
Figures \& Legends

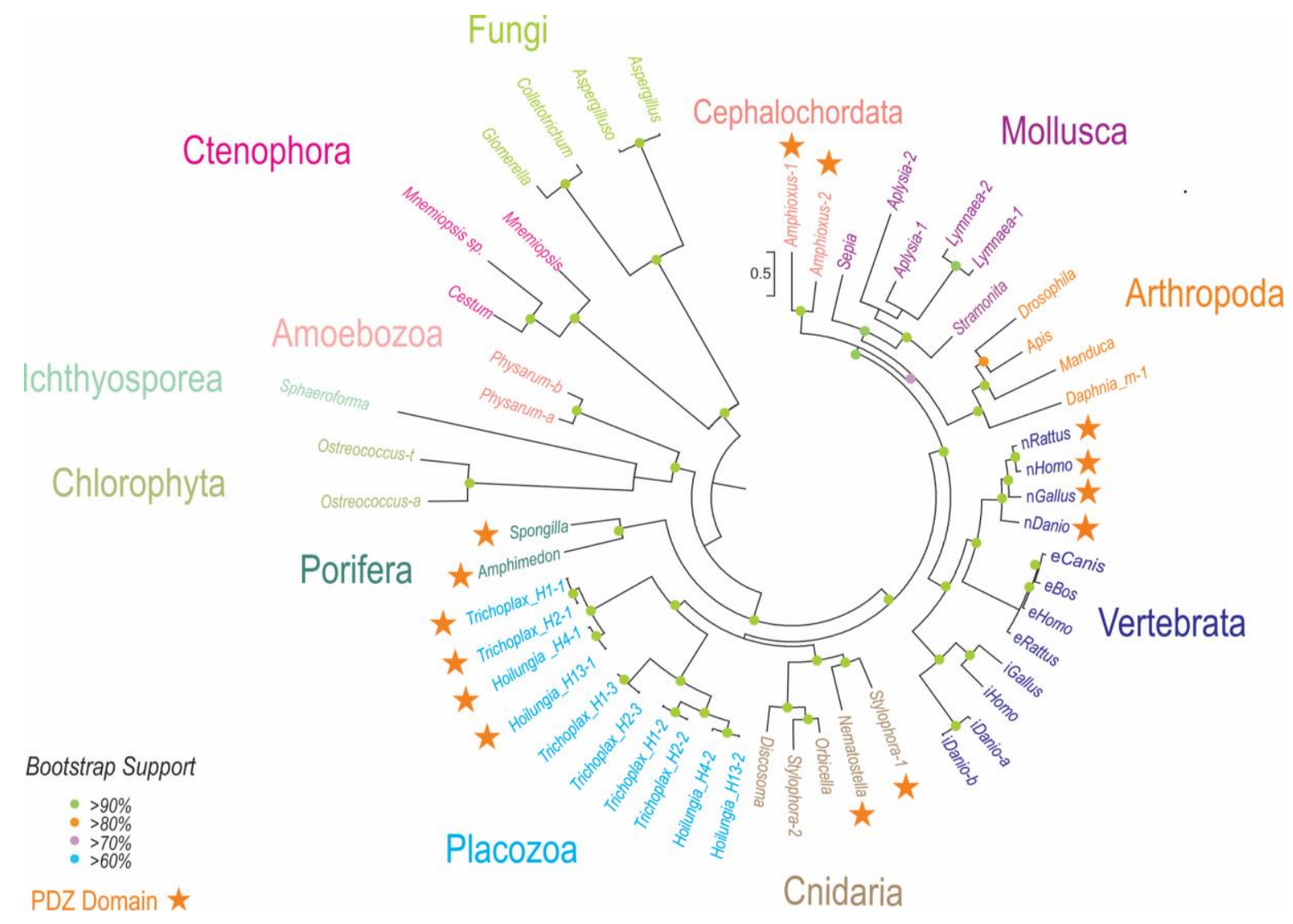

Figure 1. The diversity and evolutionary relationships of nitric oxide synthases in animals.

The representative lineages of deuterostomes, protostomes, and basal metazoans are highlighted with unicellular eukaryotes and algae as outgroups. Names of the species are indicated in each case with the existing classification of NOSs (see text for details). The references for each particular gene with relevant gene accession numbers are summarized in the method section and supplementary Table 1. 


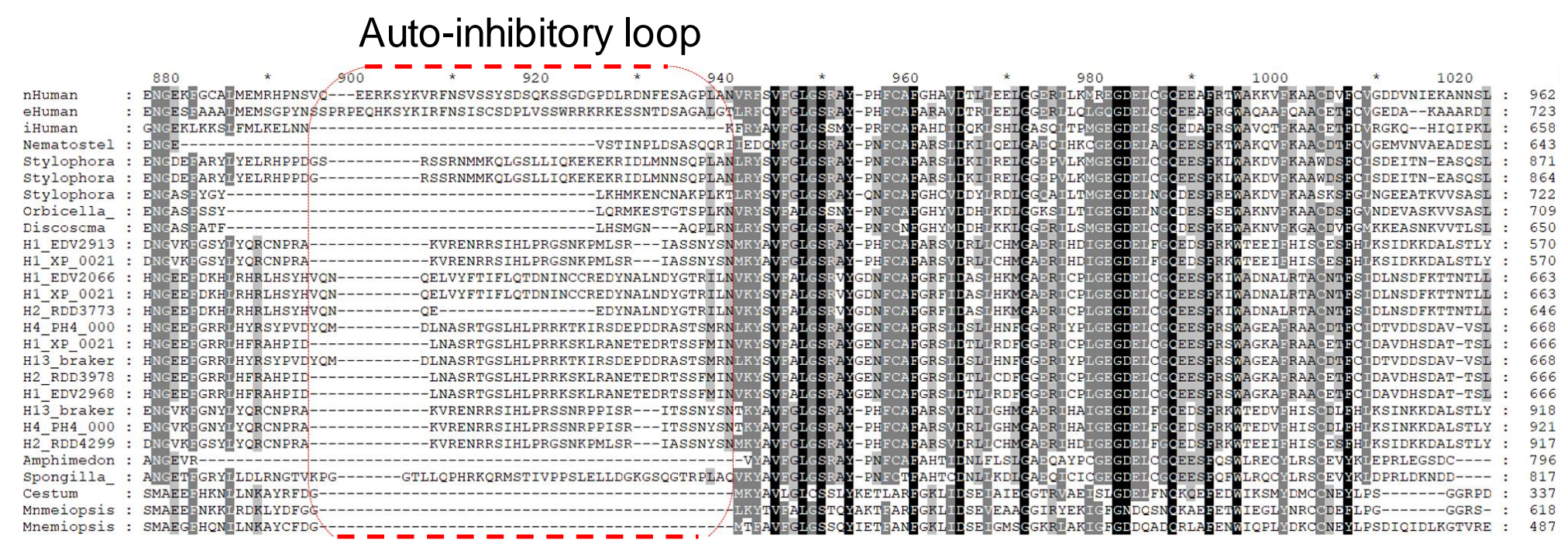

Figure 2. Auto-inhibitory N-terminal inserts in NOSs: Insights into controlling $\mathrm{Ca}^{2+}-$ dependence and the conservation of calmodulin-binding sites in placozoans. Alignments of selected NOSs was performed using the sequence information from species outlined in Fig. 1. 

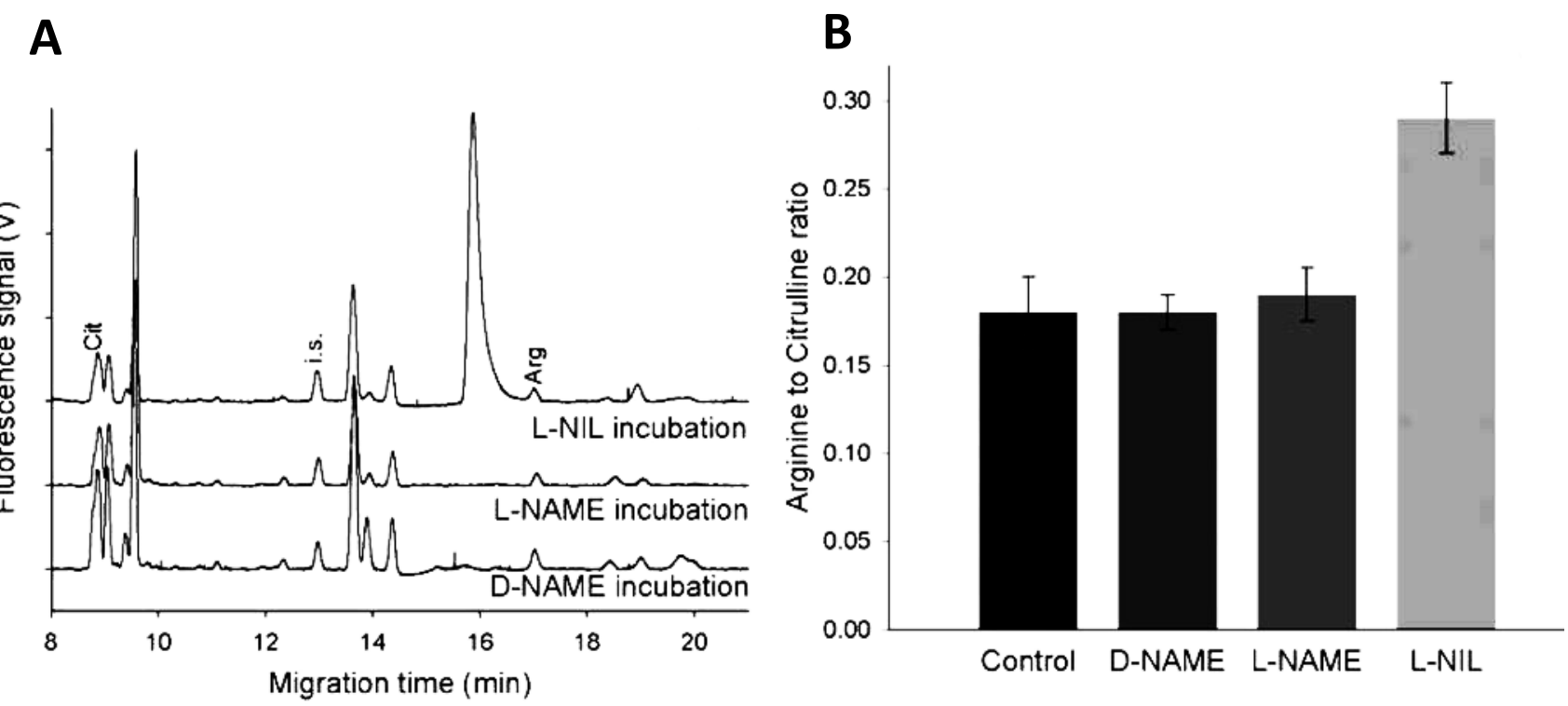

Figure 3. Detection of NOS amino acid-derived metabolites by capillary electrophoresis and their sensitivity to NOS inhibitors. A. Electropherograms of individual animal samples and LArginine to L-Citrulline ratios of Trichoplax adhaerens following treatment with NOS inhibitors. Arginine and citrulline peaks were identified with spike standards and shown as Arg and Cit, respectively. i.s. is internal standards (see methods for details). Samples were loaded using electrokinetic injection ( $8 \mathrm{kV}$ for $12 \mathrm{~s}$ ) and then analyzed under a stable $20 \mathrm{kV}$ voltage at $20^{\circ} \mathrm{C}$ in $50 \mu \mathrm{m}$ I.D. and $360 \mu \mathrm{m}$ O.D. capillary with $30 \mathrm{mM}$ borate $/ 30 \mathrm{mM}$ SDS, pH 10.0. (A) Electropherograms of Trichoplax incubated with $N^{\mathrm{G}}$-nitro-l-arginine methyl ester or L-NAME $(500 \mu \mathrm{M})$, D-NAME $(500 \mu \mathrm{M})$, and L-N ${ }^{6}$-(1-iminoethyl)-lysine, L-NIL $(1 \mathrm{mM})$, for $30 \mathrm{~min}$ at room temperature. B. Arginine-to-Citrulline ratio of Trichoplax after treatment with putative NOS inhibitors; only L-NIL induced statistically significant increase of $\mathrm{Arg} / \mathrm{Cit}$ ration suggesting the suppression of L-citrulline production ( $n=5, p<0.05$, see results for details). 
A

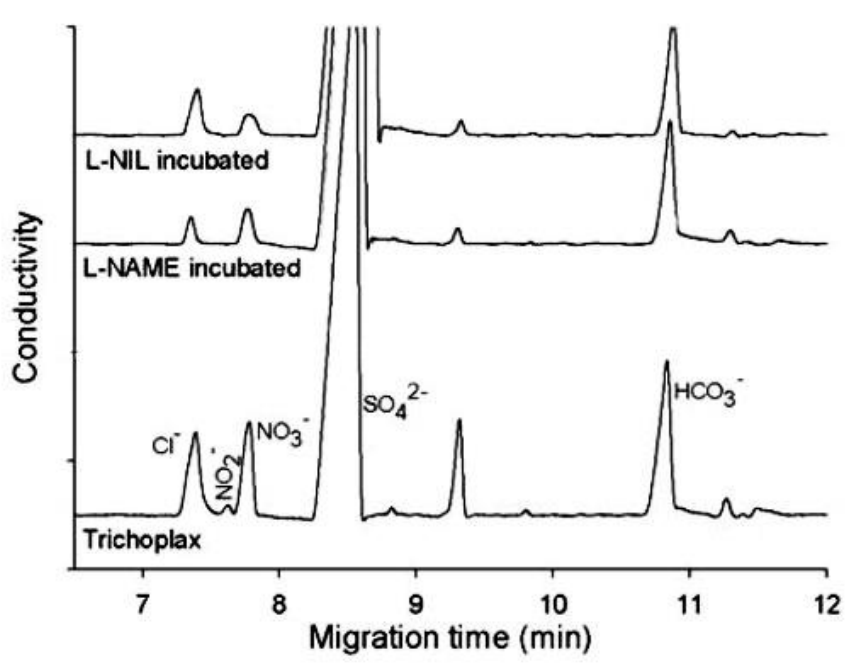

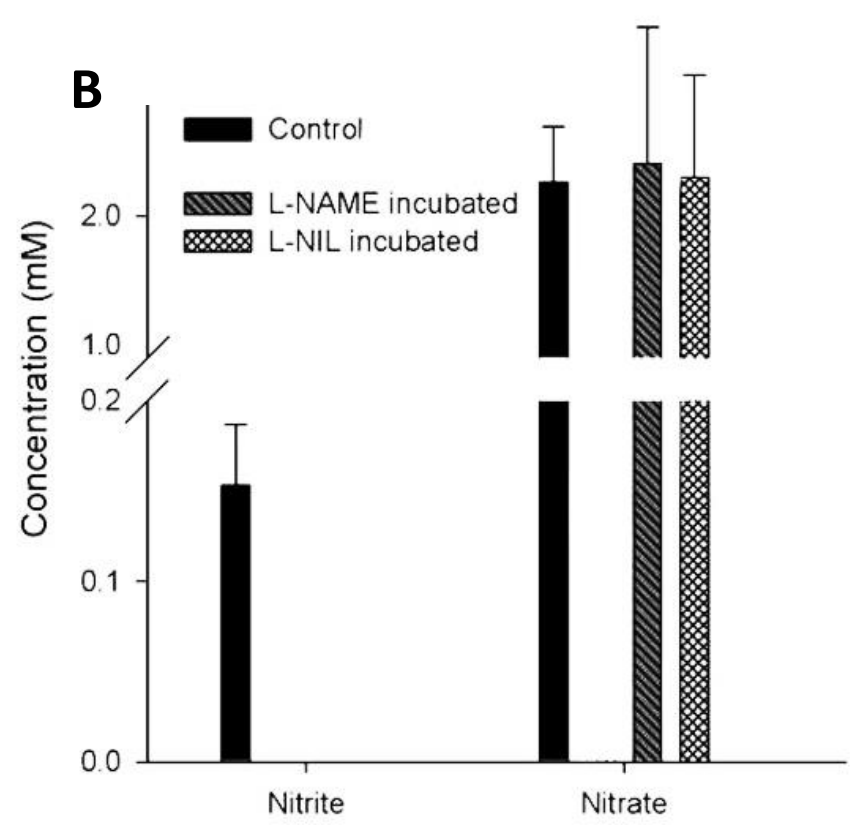

Figure 4. Detection of products of $\mathrm{NO}$ oxidation $\left(\mathrm{NO}_{2}^{-}\right.$and $\left.\mathrm{NO}_{3}^{-}\right)$by capillary electrophoresis and their sensitivity to NOS inhibitors. Nitrites, products of NO oxidation, were detected in all control samples and eliminated following NOS inhibitor incubation (see text for details). The separation was conducted in a $75 \mathrm{~cm}$ length of $50 \mu \mathrm{m}$ I.D. and $360 \mu \mathrm{m}$ O.D. capillary with arginine/borate buffer, $\mathrm{pH}$ 9.0. All samples were loaded using electrokinetic injection (-1 kV for $12 \mathrm{~s}$ ), and then analyzed under a stable $-15 \mathrm{kV}$ voltage at $20^{\circ} \mathrm{C}$. A. Electropherograms of Trichoplax only, and Trichoplax incubated for 30 mins with $N^{\mathrm{G}}$-nitro-l-arginine methyl ester or L-NAME (500 $\mu \mathrm{M})$, and L-N $\mathrm{N}^{6}-(1$-iminoethyl)-lysine or L-NIL $(1 \mathrm{mM})$. B. Nitrite and nitrate concentration profiling after 30 mins of NOS inhibition $(n=5, p<0.05)$. 

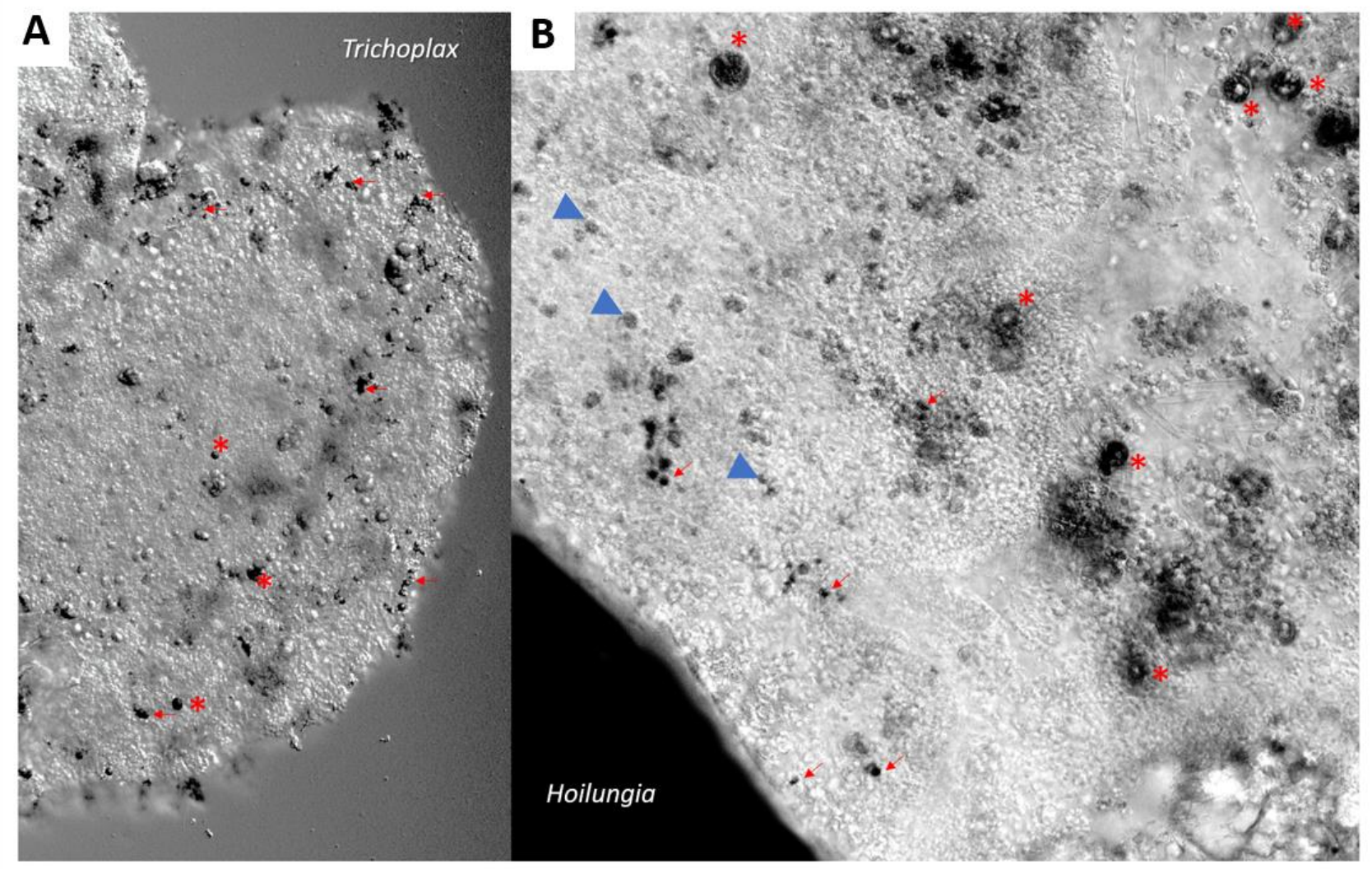

\section{C}

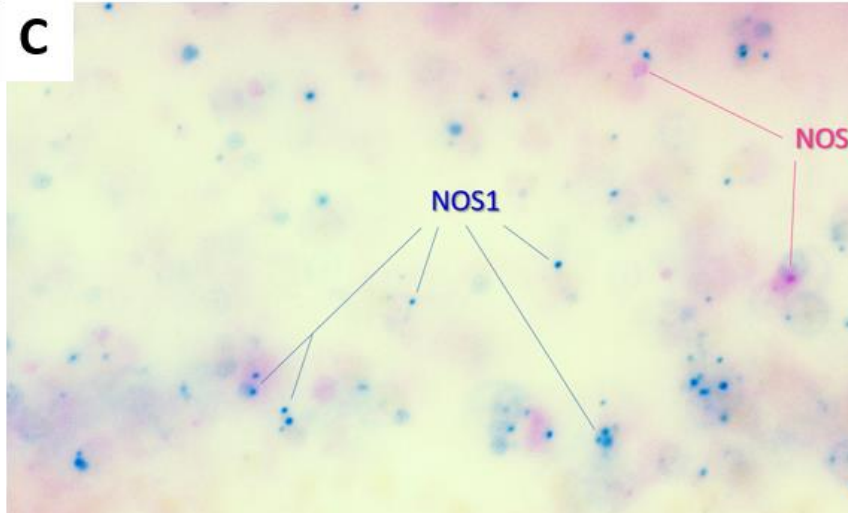

Peripheral (edge) area

$10 \mu \mathrm{m}$

Figure 5. NOS expression in Placozoans. (A, B) NADPH-diaphorase histochemistry and the distribution of putative nitrergic cells in two species of Placozoa: Trichoplax adhaerens $(\mathbf{A})$ and Hoilungia hongkongensis (B). NADPH-d reactive cells (black) are broadly distributed across the animal. In both species, relatively large cells (asterisks) correspond to so-called "shiny spheres," whereas the arrows indicate an example of NADPH-d reactive cells with some tendencies of their distribution close to the edge of animals. (C) Expression of two NOSs in Trichoplax using single molecules fluorescent in situ hybridization (FISH). Blue dots - PDZ-containing NOS1 and purple dots - NOS3. A dotted circle indicates an example of a cell where both NOS are co-localized. Note, NOS-expressed cells do not occur at the very edge of the animal. Scale: $10 \mu \mathrm{m}$. 
A

Guanylate cyclases in Placozoa

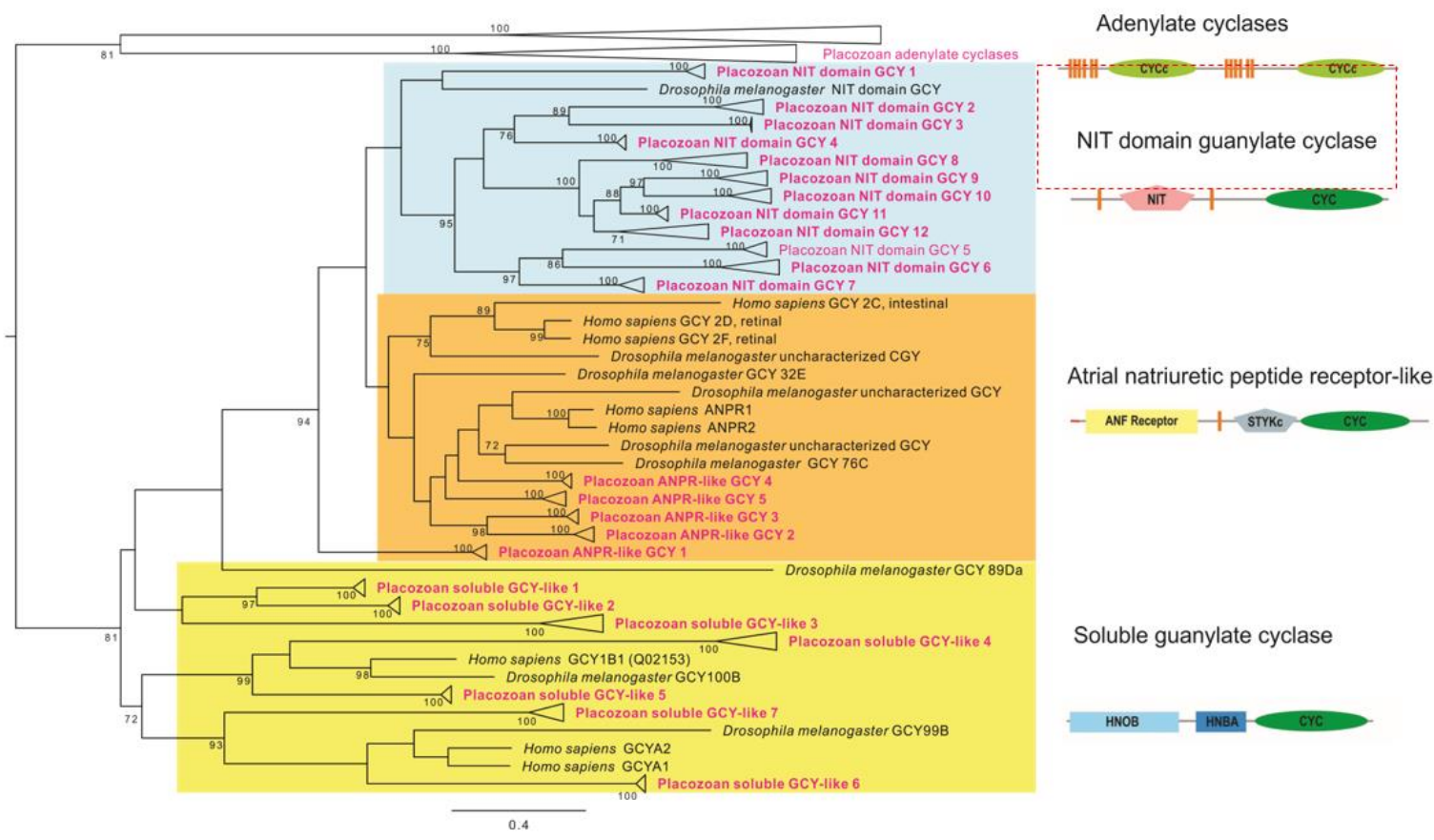

B

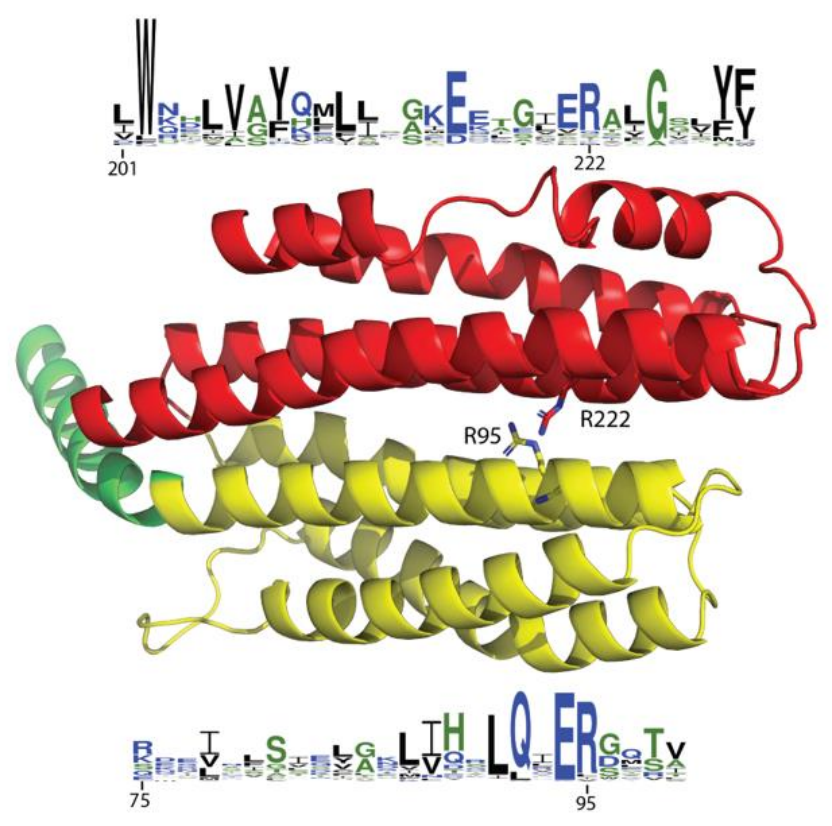

NIT domain in Trichoplax

Figure 6. The diversity and lineage-specific expansion of $\mathrm{sGC}$ and related NO receptors in placozoans. A. Maximum likelihood phylogenetic tree of placozoan soluble guanylyl cyclases (sGC) and two groups of related enzymes: Atrial Natriuretic Peptide-like receptors (ANPRs), some of which contain unusual NIT domains - putative nitrite/nitrate sensing receptors (see text), and adenylate cyclases as outgroups. 119 protein sequences (Supplementary Table 1) were trimmed 
down to cyclase domains and produced an alignment 325 aa long. Alignment was analyzed in IQTREE ${ }^{92}$ using LG+I+G4 evolution model chosen automatically with Bayesian information criterion. Tree robustness was tested with 2000 replicates of ultrafast bootstrap. Orthologous proteins from 4 placozoan species (red text) were analyzed and their branches collapsed in the tree: Trichoplax adhaerens (H1), Trichoplax sp. (H2), Hoilungia sp. (H4) and Hoilungia hongkongensis (H13), except for adenylate cyclases that were only from Trichoplax adhaerens, and NIT domain GCY3 which were found only in Hoilungia genus. Human and Drosophila orthologs are shown. The domain organization of three groups of predicted guanylyl cyclases in placozoans is also schematically illustrated. Full (uncollapsed) version of this tree can be found in Supplemental Figure 1S.

B. NIT domains in placozoans. The putative nitrate- and nitrite-sensing NIT domains of animals are homologous to prokaryotic NIT domains. Phyre2 was used to generate a structural model for the NIT domain of 007393 from Trichoplax adhaerens. The Phyre model is mostly based on the structure NIT domain of the NasR transcription antiterminator (pdb ID: 4AKK). The NIT domain consists of two four-helix bundles, shown in yellow and red. At their interface, two conserved arginines are thought to be involved in ligand binding. The sequence conservation of the two helices at the interface is shown by a webLogo representation ${ }^{93}$. The overall height of a stack indicates the sequence conservation at a certain position, whereas the height of symbols within the stack indicates the relative frequency of each amino acid at that position. 
bioRxiv preprint doi: https://doi.org/10.1101/2020.04.10.034207; this version posted April 12, 2020. The copyright holder for this preprint (which was not certified by peer review) is the author/funder, who has granted bioRxiv a license to display the preprint in perpetuity. It is made available under aCC-BY-NC-ND 4.0 International license.

\section{Supporting Information}

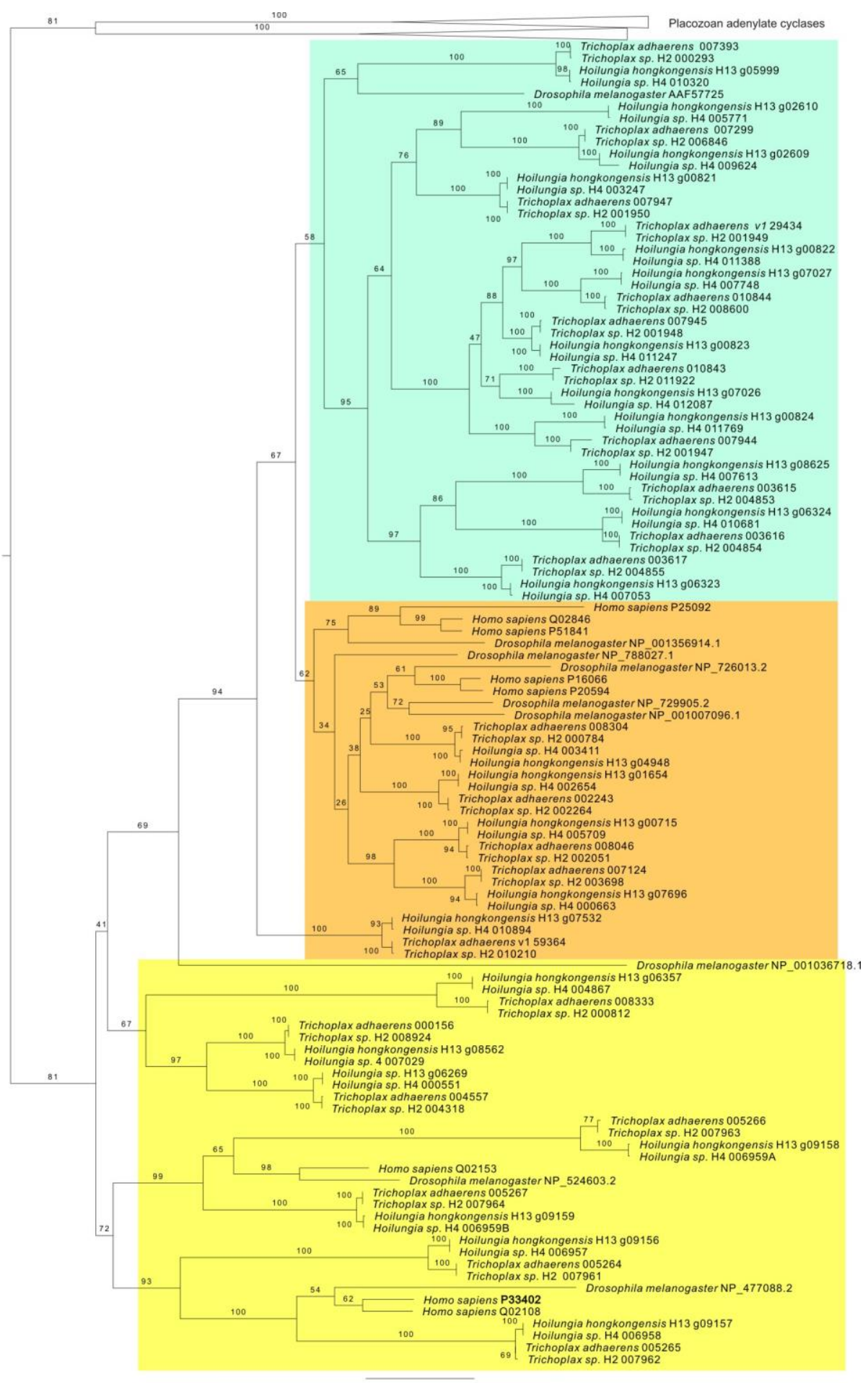

Figure 1S. Maximum likelihood phylogenetic tree of placozoan soluble guanylyl cyclases (sGC) and two groups of related enzymes: Atrial Natriuretic Peptide-like receptors (ANPRs), some of which contain unusual NIT domains, and adenylate cyclases. Proteins from all four placozoan species used in this study are represented (see Fig 6A in the main text). 


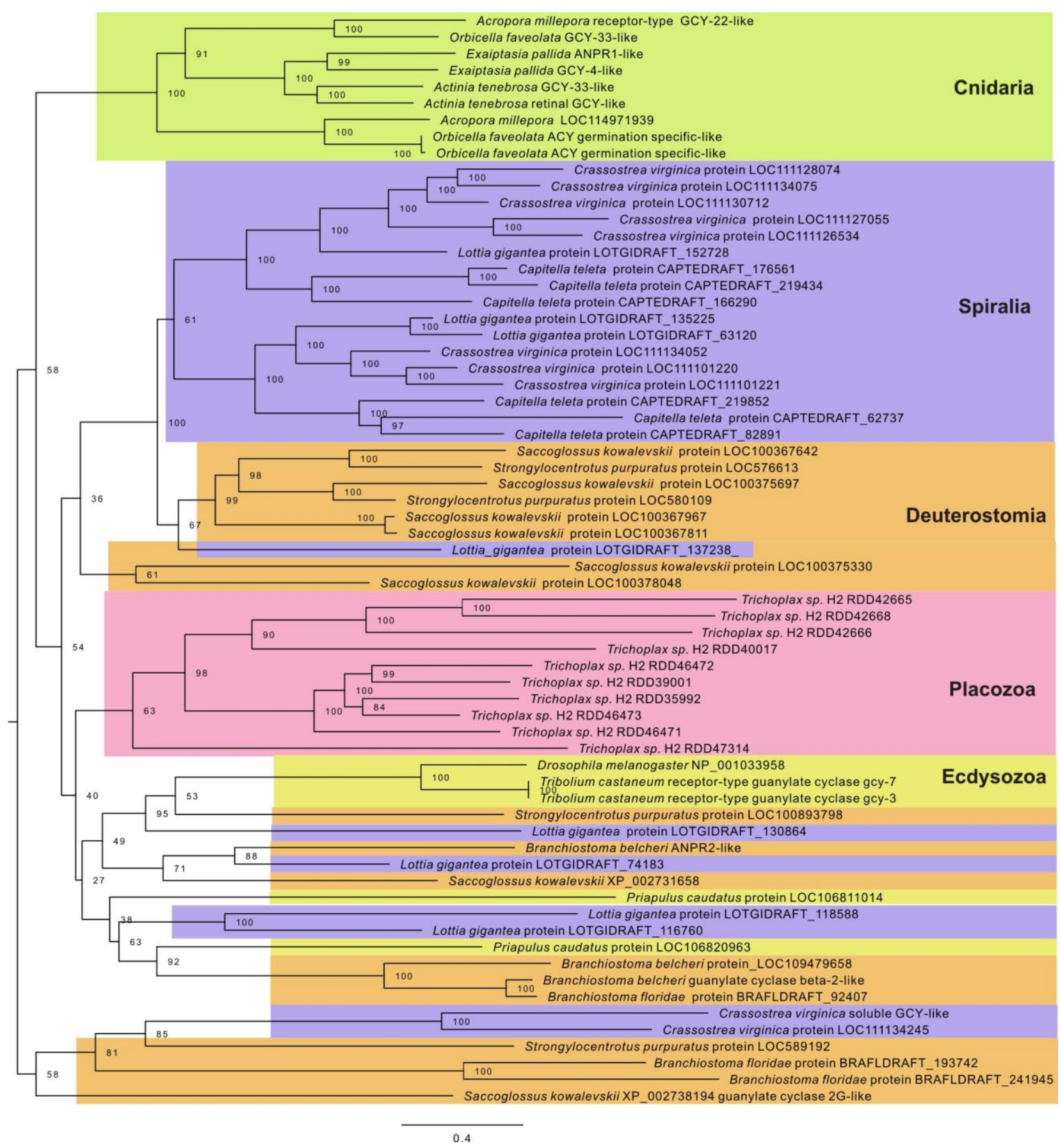

Figure 2S. Maximum likelihood phylogenetic tree of NIT domains containing guanylyl cyclases in placozoans (Trichoplax sp. $\mathrm{H} 2$ only), cnidarians and bilaterians. Guanylate cyclases with NIT domains are found in most animal phyla except sponges, ctenophores, vertebrates and urochordates. Extensive lineage-specific duplications are evident in placozoans, molluscs and hemichordates. 66 protein sequences were trimmed down to NIT+cyclase domains and produced an alignment 689 aa long. Alignment was analyzed in IQTREE ${ }^{92}$ using LG+F+R6 evolution model chosen automatically with Bayesian information criterion. Tree robustness was tested with 2000 replicates of ultrafast bootstrap. 


\section{References}

$1 \quad$ Moroz, L. L. \& Kohn, A. B. Parallel evolution of nitric oxide signaling: diversity of synthesis and memory pathways. Front Biosci (Landmark Ed) 16, 2008-2051, doi:10.2741/3837 (2011).

2 Astier, J., Mounier, A., Santolini, J., Jeandroz, S. \& Wendehenne, D. The evolution of nitric oxide signalling diverges between animal and green lineages. $J$ Exp Bot 70, 4355-4364, doi:10.1093/jxb/erz088 (2019).

3 Santolini, J. What does "NOSynthase" stand for ? Front Biosci (Landmark Ed) 24, 133-171 (2019).

4 Moncada, S., Palmer, R. M. \& Higgs, E. A. Nitric oxide: physiology, pathophysiology, and pharmacology. Pharmacol Rev 43, 109-142 (1991).

$5 \quad$ Ignarro, L. J. 1003 (Academic Press, San Diego, 2000).

6 Krumenacker, J. S., Hanafy, K. A. \& Murad, F. Regulation of nitric oxide and soluble guanylyl cyclase. Brain Res Bull 62, 505-515, doi:10.1016/S0361-9230(03)00102-3 (2004).

7 Martin, E., Berka, V., Tsai, A. L. \& Murad, F. Soluble guanylyl cyclase: the nitric oxide receptor. Methods Enzymol 396, 478-492, doi:10.1016/S0076-6879(05)96040-0 (2005).

8 Horst, B. G. \& Marletta, M. A. Physiological activation and deactivation of soluble guanylate cyclase. Nitric Oxide 77, 65-74, doi:10.1016/j.niox.2018.04.011 (2018).

9 Zhou, L. \& Zhu, D. Y. Neuronal nitric oxide synthase: structure, subcellular localization, regulation, and clinical implications. Nitric Oxide 20, 223230, doi:10.1016/j.niox.2009.03.001 (2009).
10 Colasanti, M., Venturini, G., Merante, A., Musci, G. \& Lauro, G. M. Nitric oxide involvement in Hydra vulgaris very primitive olfactory-like system. J Neurosci 17, 493-499 (1997).

11 Cristino, L., Guglielmotti, V., Cotugno, A., Musio, C. \& Santillo, S. Nitric oxide signaling pathways at neural level in invertebrates: functional implications in cnidarians. Brain Res 1225, 17-25, doi:10.1016/j.brainres.2008.04.056 (2008).

12 Colasanti, M., Mazzone, V., Mancinelli, L., Leone, S. \& Venturini, G. Involvement of nitric oxide in the head regeneration of Hydra vulgaris. Nitric Oxide 21, 164170, doi:10.1016/j.niox.2009.07.003 (2009).

13 Moroz, L. L., Meech, R. W., Sweedler, J. V. \& Mackie, G. O. Nitric oxide regulates swimming in the jellyfish Aglantha digitale. $J$ Comp Neurol 471, 26-36, doi:10.1002/cne.20023 (2004).

14 Ueda, N. et al. An ancient role for nitric oxide in regulating the animal pelagobenthic life cycle: evidence from a marine sponge. Sci Rep 6, 37546, doi:10.1038/srep37546 (2016).

15 Say, T. E. \& Degnan, S. M. Molecular and behavioural evidence that interdependent photo - and chemosensory systems regulate larval settlement in a marine sponge. Mol Ecol, doi:10.1111/mec.15318 (2019).

16 Ellwanger, K. \& Nickel, M. Neuroactive substances specifically modulate rhythmic body contractions in the nerveless metazoon Tethya wilhelma (Demospongiae, Porifera). Front Zool 3, 7, doi:10.1186/17429994-3-7 (2006). 
17 Moroz, L. L. \& Kohn, A. B. Independent origins of neurons and synapses: insights from ctenophores. Philos Trans $R$ Soc Lond B Biol Sci 371, 20150041, doi:10.1098/rstb.2015.0041 (2016).

18 Moroz, L. L. et al. The ctenophore genome and the evolutionary origins of neural systems. Nature 510, 109114, doi:10.1038/nature13400 (2014).

19 Whelan, N. V. et al. Ctenophore relationships and their placement as the sister group to all other animals. Nat Ecol Evol 1, 1737-1746, doi:10.1038/s41559-017-0331-3 (2017).

20 Laumer, C. E. et al. Revisiting metazoan phylogeny with genomic sampling of all phyla. Proc Biol Sci 286, 20190831, doi:10.1098/rspb.2019.0831 (2019).

21 Laumer, C. E. et al. Support for a clade of Placozoa and Cnidaria in genes with minimal compositional bias. Elife 7, doi:10.7554/eLife.36278 (2018).

22 Varoqueaux, F. \& Fasshauer, D. Getting Nervous: An Evolutionary Overhaul for Communication. Annu Rev Genet 51, 455-476, doi:10.1146/annurev-genet-120116024648 (2017).

23 Schierwater, B. \& DeSalle, R. Placozoa. Curr Biol 28, R97-R98, doi:10.1016/j.cub.2017.11.042 (2018).

24 Smith, C. L. et al. Novel cell types, neurosecretory cells, and body plan of the early-diverging metazoan Trichoplax adhaerens. Curr Biol 24, 1565-1572, doi:10.1016/j.cub.2014.05.046 (2014).

25 Armon, S., Bull, M. S., Aranda-Diaz, A. \& Prakash, M. Ultrafast epithelial contractions provide insights into contraction speed limits and tissue integrity. Proc Natl Acad Sci U S A 115, E10333-E10341, doi:10.1073/pnas.1802934115 (2018).

26 Smith, C. L., Reese, T. S., Govezensky, T. \& Barrio, R. A. Coherent directed movement toward food modeled in Trichoplax, a ciliated animal lacking a nervous system. Proc Natl Acad Sci U S A 116, 8901-8908, doi:10.1073/pnas.1815655116 (2019).

27 Varoqueaux, F. et al. High Cell Diversity and Complex Peptidergic Signaling Underlie Placozoan Behavior. Curr Biol 28, 3495-3501 e3492, doi:10.1016/j.cub.2018.08.067 (2018).

28 Smith, C. L., Pivovarova, N. \& Reese, T. S. Coordinated Feeding Behavior in Trichoplax, an Animal without Synapses. PLoS One 10, e0136098, doi:10.1371/journal.pone.0136098 (2015).

29 Fortunato, A. \& Aktipis, A. Social feeding behavior of Trichoplax adhaerens. Front Ecol Evol 7, doi:10.3389/fevo.2019.00019 (2019).

30 Heyland, A., Croll, R., Goodall, S., Kranyak, J. \& Wyeth, R. Trichoplax adhaerens, an enigmatic basal metazoan with potential. Methods Mol Biol 1128, 45-61, doi:10.1007/978-1-62703-974-1_4 (2014).

31 Cruz, L., Moroz, L. L., Gillette, R. \& Sweedler, J. V. Nitrite and nitrate levels in individual molluscan neurons: single-cell capillary electrophoresis analysis. $J$ Neurochem 69, 110-115, 
doi:10.1046/j.1471-

4159.1997.69010110.x (1997).

32 Floyd, P. D., Moroz, L. L., Gillette, R. \& Sweedler, J. V. Capillary electrophoresis analysis of nitric oxide synthase related metabolites in single identified neurons. Anal Chem 70, 2243-2247, doi:10.1021/ac9713013 (1998).

33 Moroz, L. L., Dahlgren, R. L., Boudko, D., Sweedler, J. V. \& Lovell, P. Direct single cell determination of nitric oxide synthase related metabolites in identified nitrergic neurons. J Inorg Biochem 99, 929939,

doi:10.1016/j.jinorgbio.2005.01.013 (2005).

34 Boudko, D. Y., Cooper, B. Y., Harvey, W. R. \& Moroz, L. L. Highresolution microanalysis of nitrite and nitrate in neuronal tissues by capillary electrophoresis with conductivity detection. J Chromatogr B Analyt Technol Biomed Life Sci 774, 97-104, doi:10.1016/s1570-0232(02)00219-2 (2002).

35 Bodnarova, M., Martasek, P. \& Moroz, L. L. Calcium/calmodulindependent nitric oxide synthase activity in the CNS of Aplysia californica: biochemical characterization and link to cGMP pathways. J Inorg Biochem 99, 922928,

doi:10.1016/j.jinorgbio.2005.01.012 (2005).

36 Eitel, M. et al. Comparative genomics and the nature of placozoan species. PLoS Biol 16, e2005359, doi:10.1371/journal.pbio.2005359 (2018).

37 Srivastava, M. et al. The Trichoplax genome and the nature of placozoans. Nature $\quad 454, \quad 955-960$, doi:10.1038/nature07191 (2008).
38 Edgar, R. MUSCLE: multiple sequence alignment with high accuracy and high throughput. Nucleic Acids Res 32, 1792-1797 ( 2004).

39 Stamatakis, A. RAxML version 8: a tool for phylogenetic analysis and post-analysis of large phylogenies. Bioinformatics (Oxford, England) 30, 1312-1313 (2014).

40 Ronquist, F. et al. MrBayes 3.2: efficient Bayesian phylogenetic inference and model choice across a large model space. Systematic Biology 61, 539-542 (2012).

41 Rambaut, A., Drummond, A. J., Xie, D., Baele, G. \& Suchard, M. A. Posterior summarisation in Bayesian phylogenetics using Tracer 1.7. . Systematic Biology 67, 901-904 (2018).

42 Kumar, S., Stecher, G., Li, M., Knyaz, C. \& Tamura, K. MEGA X: Molecular Evolutionary Genetics Analysis across computing platforms. Mol. Biol. Evol. 35, 1547-1549 (2018).

43 Nei, M. \& Gojobori, T. Simple methods for estimating the numbers of synonymous and nonsynonymous nucleotide substitutions. Mol. Biol. Evol. 3, 418-426 (1986).

44 Zhang, J., Kumar, S. \& Nei, M. Small-sample tests of episodic adaptive evolution: a case study of primate lysozymes. : Mol. Biol. Evol 14, 1335-1338 (1997).

45 Kosakovsky Pond, S. L., Frost, S. D. \& Muse, S. V. HyPhy: hypothesis testing using phylogenies. . Bioinformatics 21, 676-679 (2005).

46 Murrell, B. et al. FUBAR: A Fast, Unconstrained Bayesian AppRoximation for Inferring Selection. Mol. Biol. Evol. 30, 11961205 (2013). 
47 Murrell, B. et al. Gene-wide identification of episodic selection. Molecular Biology and Evolution 32, 1365-1371 (2015).

48 Murrell, B. et al. Detecting Individual Sites Subject to Episodic Diversifying Selection. . PLoS Genetics 8, e1002764. (2012).

49 Smith, M. D. et al. Less Is More: An adaptive branch-site random effects model for efficient detection of episodic diversifying selection. Mol. Biol. Evol. 32, 1342-1353 ( 2015).

50 Moroz, L. L., Gillette, R. \& Sweedler, J. V. Single-cell analyses of nitrergic neurons in simple nervous systems. $J$ Exp Biol 202, 333-341 (1999).

51 Dawson, T. M., Bredt, D. S., Fotuhi, M., Hwang, P. M. \& Snyder, S. H. Nitric oxide synthase and neuronal NADPH diaphorase are identical in brain and peripheral tissues. Proc Natl Acad Sci U S A 88, 7797-7801, doi:10.1073/pnas.88.17.7797 (1991).

52 Hope, B. T., Michael, G. J., Knigge, K. M. \& Vincent, S. R. Neuronal NADPH diaphorase is a nitric oxide synthase. Proc Natl Acad Sci U S A 88, 2811-2814, doi:10.1073/pnas.88.7.2811 (1991).

53 Pasqualotto, B. A., Hope, B. T. \& Vincent, S. R. Citrulline in the rat brain: immunohistochemistry and coexistence with NADPHdiaphorase. Neurosci Lett 128, 155160, doi:10.1016/03043940(91)90250-w (1991).

54 Kurenni, D. E. et al. Nitric oxide synthase in tiger salamander retina. $J$ Comp Neurol 361, 525-536, doi:10.1002/cne.903610314 (1995).

55 Moroz, L. L. Giant identified NOreleasing neurons and comparative histochemistry of putative nitrergic systems in gastropod molluscs. Microsc Res Tech 49, 557-569, doi:10.1002/1097-

0029(20000615)49:6<557::AID-

JEMT6>3.0.CO;2-S (2000).

56 Moroz, L. L., Norekian, T. P., Pirtle, T. J., Robertson, K. J. \& Satterlie, R. A. Distribution of NADPHdiaphorase reactivity and effects of nitric oxide on feeding and locomotory circuitry in the pteropod mollusc, Clione limacina. J Comp Neurol 427, 274-284 (2000).

57 Turner, R. W. \& Moroz, L. L. Localization of nicotinamide adenine dinucleotide phosphate-diaphorase activity in electrosensory and electromotor systems of a gymnotiform teleost, Apteronotus leptorhynchus. J Comp Neurol 356, 261-274, doi:10.1002/cne.903560209 (1995).

58 King, N. et al. The genome of the choanoflagellate Monosiga brevicollis and the origin of metazoans. Nature 451, 783-788, doi:10.1038/nature06617 (2008).

59 Whelan, N. V., Kocot, K. M., Moroz, L. L. \& Halanych, K. M. Error, signal, and the placement of Ctenophora sister to all other animals. Proc Natl Acad Sci U S A 112, 57735778, doi:10.1073/pnas.1503453112 (2015).

60 Eitel, M., Osigus, H. J., DeSalle, R. \& Schierwater, B. Global diversity of the Placozoa. PLoS One 8, e57131, doi:10.1371/journal.pone.0057131 (2013).

61 Merino-Gracia, J., Costas-Insua, C., Canales, M. A. \& Rodriguez-Crespo, I. Insights into the C-terminal Peptide Binding Specificity of the PDZ Domain of Neuronal Nitric-oxide Synthase: CHARACTERIZATION OF THE INTERACTION WITH THE TIGHT JUNCTION PROTEIN CLAUDIN-3. J Biol Chem 291, 
11581-11595,

doi:10.1074/jbc.M116.724427

(2016).

62 Costas-Insua, C., Merino-Gracia, J., Aicart-Ramos, C. \& RodriguezCrespo, I. Subcellular Targeting of Nitric Oxide Synthases Mediated by Their N-Terminal Motifs. $A d v$ Protein Chem Struct Biol 111, 165195 ,

doi:10.1016/bs.apcsb.2017.07.002 (2018).

63 Manivet, P. et al. PDZ-dependent activation of nitric-oxide synthases by the serotonin 2B receptor. $J$ Biol Chem 275, 9324-9331, doi:10.1074/jbc.275.13.9324 (2000).

64 Tochio, H. et al. Formation of nNOS/PSD-95 PDZ dimer requires a preformed beta-finger structure from the nNOS PDZ domain. $J$ Mol Biol 303, 359-370, doi:10.1006/jmbi.2000.4148 (2000).

65 Hillier, B. J., Christopherson, K. S., Prehoda, K. E., Bredt, D. S. \& Lim, W. A. Unexpected modes of PDZ domain scaffolding revealed by structure of nNOS-syntrophin complex. Science 284, 812-815 (1999).

66 Nishida, C. R. \& Ortiz de Montellano, P. R. Autoinhibition of endothelial nitric-oxide synthase. Identification of an electron transfer control element. J Biol Chem 274, 1469214698, doi:10.1074/jbc.274.21.14692 (1999).

67 Salerno, J. C. et al. An autoinhibitory control element defines calciumregulated isoforms of nitric oxide synthase. J Biol Chem 272, 2976929777, doi:10.1074/jbc.272.47.29769 (1997).

68 Daff, S., Sagami, I. \& Shimizu, T. The 42-amino acid insert in the FMN domain of neuronal nitric-oxide synthase exerts control over $\mathrm{Ca}(2+) /$ calmodulin-dependent electron transfer. J Biol Chem 274, 30589-30595, doi:10.1074/jbc.274.43.30589 (1999).

69 Jones, R. J. et al. The function of the small insertion in the hinge subdomain in the control of constitutive mammalian nitric-oxide synthases. J Biol Chem 279, 3687636883, doi:10.1074/jbc.M402808200 (2004).

70 Knudsen, G. M., Nishida, C. R., Mooney, S. D. \& Ortiz de Montellano, P. R. Nitric-oxide synthase (NOS) reductase domain models suggest a new control element in endothelial NOS that attenuates calmodulin-dependent activity. J Biol Chem 278, 31814-31824, doi:10.1074/jbc.M303267200 (2003).

71 Lane, P. \& Gross, S. S. The autoinhibitory control element and calmodulin conspire to provide physiological modulation of endothelial and neuronal nitric oxide synthase activity. Acta Physiol Scand 168, 53-63, doi:10.1046/j.1365201x.2000.00654.x (2000).

72 Li, D., Stuehr, D. J., Yeh, S. R. \& Rousseau, D. L. Heme distortion modulated by ligand-protein interactions in inducible nitric-oxide synthase. J Biol Chem 279, 2648926499, doi:10.1074/jbc.M400968200 (2004).

73 Nishida, C. R. \& de Montellano, P. R. Control of electron transfer in nitricoxide synthases. Swapping of autoinhibitory elements among nitricoxide synthase isoforms. $\mathrm{J} \mathrm{Biol} \mathrm{Chem}$ 276, 20116-20124, doi:10.1074/jbc.M101548200 (2001). 
74 Moroz, L. L. et al. Non-enzymatic production of nitric oxide (NO) from NO synthase inhibitors. Biochem Biophys Res Commun 253, 571-576, doi:10.1006/bbrc.1998.9810 (1998).

75 Moroz, L. L. \& Gillette, R. From Polyplacophora to Cephalopoda: comparative analysis of nitric oxide signalling in mollusca. Acta Biol Hung 46, 169-182 (1995).

76 Moroz, L. L. Localization of putative nitrergic neurons in peripheral chemosensory areas and the central nervous system of Aplysia californica. J Comp Neurol 495, 1020, doi:10.1002/cne.20842 (2006).

77 Leake, L. D. \& Moroz, L. L. Putative nitric oxide synthase (NOS)containing cells in the central nervous system of the leech, Hirudo medicinalis: NADPH-diaphorase histochemistry. Brain Res 723, 115124 , doi:10.1016/00068993(96)00220-x (1996).

78 Jackson, A. M. \& Buss, L. W. Shiny spheres of placozoans (Trichoplax) function in anti-predator defense. Invertebrate Biology 128, 205-212 (2009).

79 Bassler, J., Schultz, J. E. \& Lupas, A. N. Adenylate cyclases: Receivers, transducers, and generators of signals. Cell Signal 46, 135-144, doi:10.1016/j.cellsig.2018.03.002 (2018).

80 Gancedo, J. M. Biological roles of cAMP: variations on a theme in the different kingdoms of life. Biol Rev Camb Philos Soc 88, 645-668, doi:10.1111/brv.12020 (2013).

81 Derbyshire, E. R. \& Marletta, M. A. Structure and regulation of soluble guanylate cyclase. Annu Rev Biochem 81, 533-559, doi:10.1146/annurevbiochem-050410-100030 (2012).
82 Gunn, A., Derbyshire, E. R., Marletta, M. A. \& Britt, R. D. Conformationally distinct fivecoordinate heme-NO complexes of soluble guanylate cyclase elucidated by multifrequency electron paramagnetic resonance (EPR). Biochemistry 51, 8384-8390, doi:10.1021/bi300831m (2012).

83 Montfort, W. R., Wales, J. A. \& Weichsel, A. Structure and Activation of Soluble Guanylyl Cyclase, the Nitric Oxide Sensor. Antioxid Redox Signal 26, 107-121, doi:10.1089/ars.2016.6693 (2017).

84 Horst, B. G. et al. Allosteric activation of the nitric oxide receptor soluble guanylate cyclase mapped by cryo-electron microscopy. Elife 8 , doi:10.7554/eLife.50634 (2019).

85 Kang, Y., Liu, R., Wu, J. X. \& Chen, L. Structural insights into the mechanism of human soluble guanylate cyclase. Nature 574, 206210, doi:10.1038/s41586-019-1584-6 (2019).

86 Ruiz-Stewart, I. et al. Guanylyl cyclase is an ATP sensor coupling nitric oxide signaling to cell metabolism. Proc Natl Acad Sci U S A 101, 37-42, doi:10.1073/pnas.0305080101 (2004).

87 Oulavallickal, T. et al. The Pseudomonas syringae pv. actinidiae chemoreceptor protein $\mathrm{F}$ (PscF) periplasmic sensor domain: cloning, purification and X-ray crystallographic analysis. Acta Crystallogr F Struct Biol Commun 73, 701-705, doi:10.1107/S2053230X17016831 (2017).

88 Camargo, A. et al. Nitrate signaling by the regulatory gene NIT2 in Chlamydomonas. Plant Cell 19, 
3491-3503, doi:10.1105/tpc.106.045922 (2007).

89 Shu, C. J., Ulrich, L. E. \& Zhulin, I. B. The NIT domain: a predicted nitrate-responsive module in bacterial sensory receptors. Trends Biochem Sci 28, 121-124, doi:10.1016/S09680004(03)00032-X (2003).

90 Boudes, M. et al. The structure of the NasR transcription antiterminator reveals a one-component system with a NIT nitrate receptor coupled to an ANTAR RNA-binding effector. $\mathrm{Mol}$ Microbiol $\quad \mathbf{8 5}, \quad 431-444$, doi:10.1111/j.13652958.2012.08111.x (2012).

91 Striedter, G. F. et al. NSF workshop report: discovering general principles of nervous system organization by comparing brain maps across species. J Comp Neurol 522, 1445-1453, doi:10.1002/cne.23568 (2014).

92 Trifinopoulos, J., Nguyen, L. T., von Haeseler, A. \& Minh, B. Q. W-IQTREE: a fast online phylogenetic tool for maximum likelihood analysis. Nucleic Acids Res 44, W232-235, doi:10.1093/nar/gkw256 (2016).

93 Crooks, G. E., Hon, G., Chandonia, J. M. \& Brenner, S. E. WebLogo: a sequence logo generator. Genome Res 14, 1188-1190, doi:10.1101/gr.849004 (2004). 